\title{
Modern and Digitalized USB Device With Extendable Memory Capacity
}

\author{
J. Nandini Meeraa, S. Devi \\ Abirami, N. Indhuja, R. Aravind \\ Dept of CSE, Final year \\ NSIT, Salem, India
}

\author{
C. Chithiraikkayalvizhi \\ Dept of ECE, Final year \\ Sri Krishna College of Engineering \\ Coimbatore, India
}

\author{
K. Rathina Kumar \\ Assistant Prof, Dept of ECE \\ Knowledge Institute of Technology \\ Salem, India
}

\begin{abstract}
This paper proposes a advance technology which is completely innovative and creative. The urge of inventing this proposal lies on the bases of the idea of making a pen drive have an extendable memory capacity with a modern and digitalized look. This device can operate without the use of a computer system or a mobile. The computerized pen drive has a display unit to display the contents of the pen drive and in-built USB slots to perform data transmission to other pen drives directly without the use of the computer system. The implementation of the extendable memory slots to the computerized pen drive makes it a modern digitalized and extendable USB device. The implementation of an operating system and a processor to the pen drive are the main challenges in this proposed system. The design of the system is done in such a way that the device is cost efficient and user friendly. The design process and the hardware, software structures of this modern and digitalized USB device with extendable memory capacity are explained in detail in this paper.
\end{abstract}

Keywords-extendable memory capacity;in-built USB slots; computerized pen drive; operating system;processor.

\section{INTRODUCTION}

This paper primarily focuses on the innovation of a new gadget which is completely useful for storage and data transfer. The core subject of this proposal is a pen drive device. The major reason for choosing it is in-spite of developments of many advanced technologies in the field data exchange and transmission mainly like internet through which we can send and receive a lot of data and files, but the usage of the pan drive devices is still dominant through the world and very common in the student and working community. It is rare to see a person making use of the computer system even in personal or official working environments without owning a pen drive in the present scenario.

There are also so many latest devices or the mediums in which storage of data can be made, for example the cloud computing offers a way in which we can store the data in the cloud area being provide for us. The Imagination of a normal pen drive with the display gives an attractive and enables to know the details of the contents inside the pen drive. The implementation of the in-built USB ports in the body of the pen drive provides a medium, through which another pen drive being connected to it directly and data transmission can be done even without the use of the computer system.

The way in which the pen drive models are categorized is normally based on the memory size like $2 \mathrm{~GB}, 4 \mathrm{~GB}$ up to $64 \mathrm{~GB}$ in today's market. The main idea of this paper is change this trend and bring into the advanced concept of the adjustable memory capacity pen drive devices.

This process allows the pen drive to have extendable slots through which additional memory cards can be inserted in order to increase the size of the memory capacity of the computerized pen drive. These are the brief introduction about the modern and digitalized USB device with extendable memory capacity and the concept implementation are described as follows.

\section{NORMAL PEN DRIVE MODELS AND ITS FEATURES}

\section{A. Look and Dependent on computer systems to operate}

The look of the normal pen drives which are being present now has no special features are as shown as follows in the figure 1. There is no display unit or in-built USB port for data transmission. It cannot operate without a computer system; completely depend on the computers for working with it.

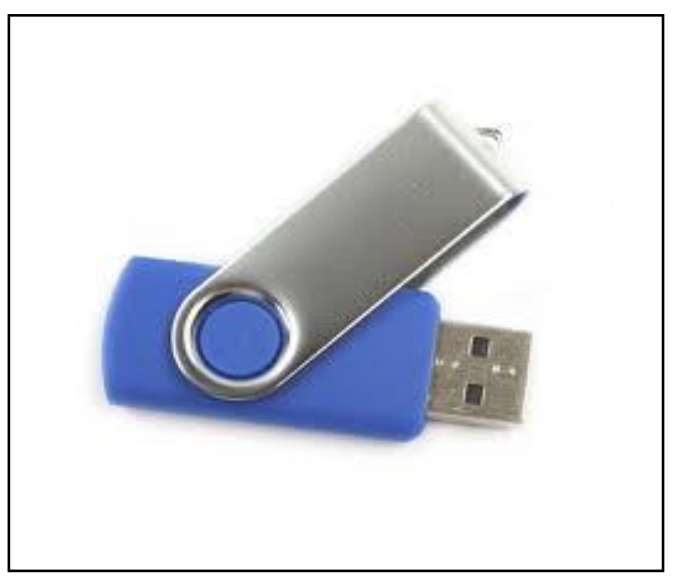

Figure 1. Look of a Normal Pen Drive.

\section{B. Fixed Memory capacity}

The normal pen drives are of fixed memory size, all the models are limited to a constant memory space. The memory capacity is a major issue while in need of extra space for storage and other operations being done through it.

So this paper provides a complete solution for this limitation and has a new approach over the sales of the pen drive which will not be on the memory size as being now. The variation in the memory can be done when there is a need for it. 


\section{COMPUTERIZED PEN DRIVE}

The look of the normal pen drives which are being present now has no special features are as shown as follows in the figure 1. There is no display unit or in-built USB port for data transmission. It cannot operate without a computer system; completely depend on the computers for working with it. Computerized Pendrive is a data storage device like text, image, video etc. and it was invented by invented by Amir Ban, Dov Moran and OronOgdan and it was established by IBM in 2000.

It is known as Universal SeriaBus (USB) interface and it does what a floppy disk dose. The essential components are A USB plug, microcontroller, flash memory chip, Crystal oscillator, Jumpers, LEDs and Write-protect switches. It supports the operating system like UNIX, LINUX, MACOS and WINDOWS.

Its main features are touch screen, two USB slots and has charge terminal it does what the entire computer does. We can transfer the data from one pen drive to the computerized pendrive and edit the data's to our needs. It is smaller, faster, cheaper and portable. It has the charge terminal to charge as like laptop's etc. They have a flash memory that is lower conception of power by advanced microprocessor. We can even play songs and videos.
The transformation of large data is done in less time. Then the appearance is very attractive and colorful. They are having a file system like,

- Defragment.

- Even Distribution.

- Hard Drive.

It is used to store the data's of booting a system ,computer forensics and law enforcement, booting operating system, windows vista and windows 7ready boost, audio player, media marketing and storage, arcades, brands and product promotions, operating system installation, medias, graphics, security systems and backup's. It is the portable devices like tape, floppy disk, optimal media, flash memory cards, external hard disk, obsolete devices, encryption and security. This computerized pendrive have a security code that we can keep a number security lock that no other people can access it. It has a touch screen ability to perform its job and know the contents of the memory in the pen drive.

There is a need of behavior analysis of flash-memory storage systems and other USB devices for storages and their evaluations. In particular, a set of evaluation metrics and their corresponding access patterns are proposed. The behaviors of flash memory are also analyzed in terms of performance and reliability issues [1].

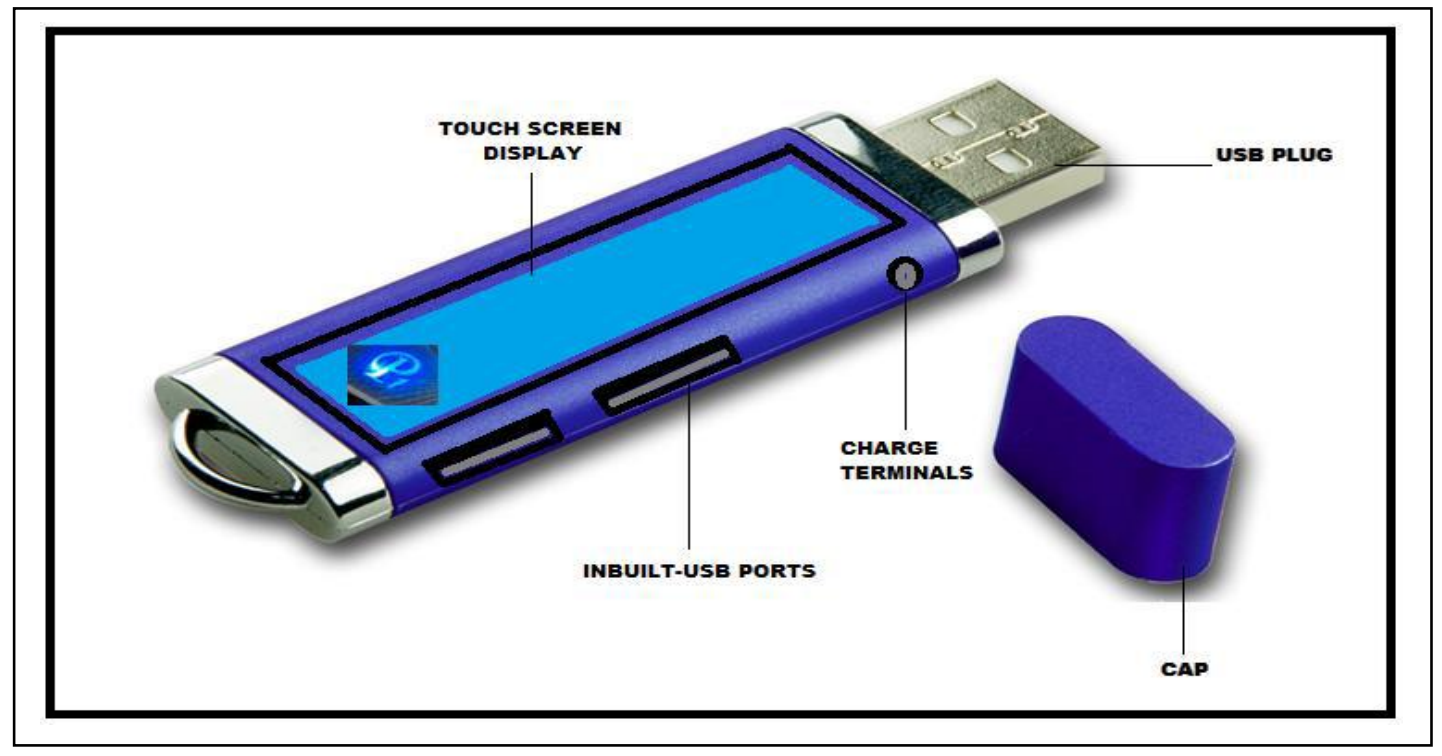

Figure 2.The computerized pen drive.

The security of the pen drive can be implemented be some techniques which can identify specific drive. We can describe the methods of digital evidence analysis [2] about USB thumb drives or devices such as Computerized Pen Drive. The rate of the pen drives which is implemented with these features can also be cheap as the normal drives being in the market now. This can really create a dynamic storage and transmission device. This proposal when implemented has more impact on the pen drive sales. The advantages of using such USB pen drives and flash drives are mainly because their power consumption and energy overhead features [3]. The main advantages are listed as follows:

- Very handy and compact

- Display unit to display its contents

- Extendable memory slots

- High speed processor and user friendly OS.

- Chargeable battery

- Affordable cost. 


\section{A. Display unit}

The display unit is the advanced feature in the modern pen drives. The display of the pen drive can be designed using touch screen .Using this unit we can see the contents in the pen drive using the touch screen. Using the touch screen in the pen drive will reduce the space occupied by the buttons in the pen drives. There are many kids of display technologies. The storage tube graphic display is the oldest technology used for display but now we are using the new virtual therapy for modern pen drives. The quality of the display can be increased by using the technology that we are using for the display. The quality of the display will be varying based on the technology we are using. We have to consider the various factors such as quality and speed while choosing the technologies that are using for display.

\section{B. In-built USB Port}

In the computerized pen drive there will be In-built USB port slot. Using that slot you can insert two pen drives in the space provided for it. This computerized pen drive is working as a computer system by providing all the features.

\section{Data Transmission}

The Data transmission is also the advanced feature in the computerized pen drive. Using this feature we can transmit the data to any other USB devices and the transmission from any other USB devices can be made very easy and simple using this computerized pen drive. The working of the computerized pen drive is similar to the computer system. The data transmission through this computerized pen drive will reduce the consumption of power. The need to connect portable electronic devices to each other has accelerated the adoption of USB onthe-go as an industry standard wired interface [4] for interfacing the two devices concepts. The data transmission in the Computerized Pen Drive can also be done without the use of the system.

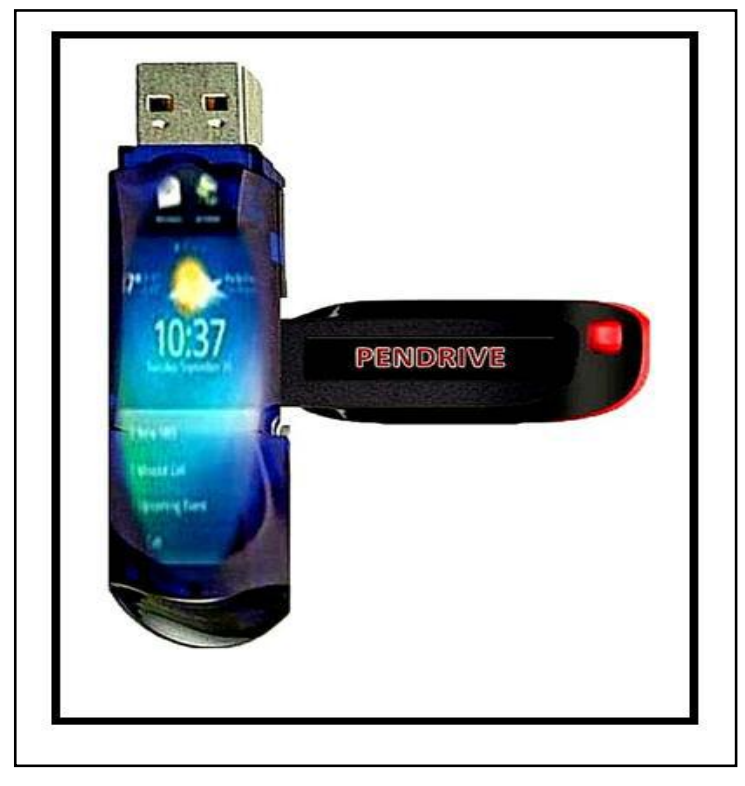

Figure 3.Data Transmission

\section{NIDR Processor}

Vol. 3, No. 11, 2012

The use of NIDR processor is used to increase the speed and the data can be transmitted in a more speed than any other processor [5]. First it will fetch the instruction and send to the identifier unit to identify the type of instruction. The multiple instruction queues are implemented to increase the temporary memory. Based on the type of instructions all the instructions will be decoded at a time and finally those instruction are send to the execute unit for execution. The operation of this processor is shown as following.

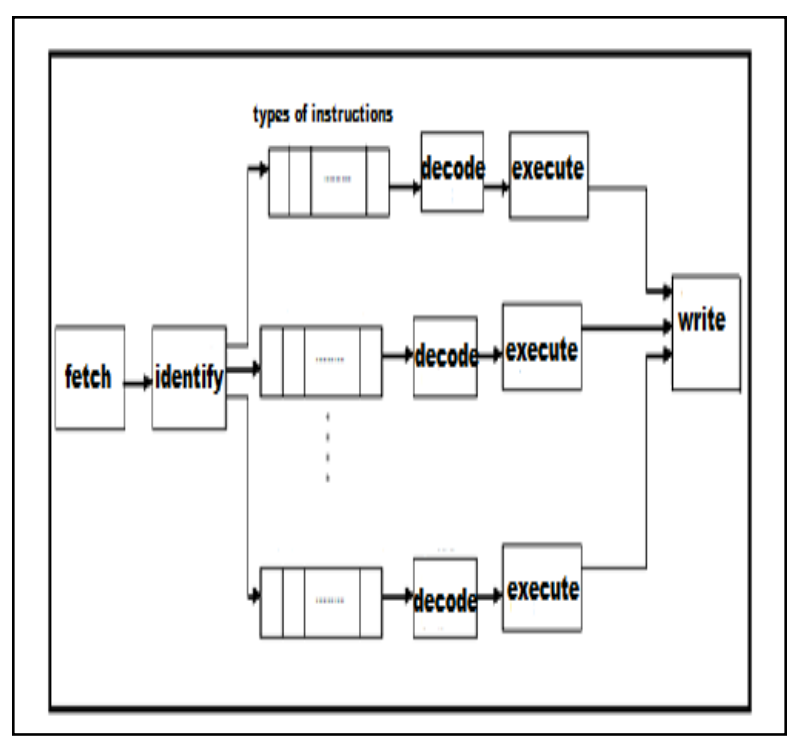

Figure 4.NIDR Processor

\section{E. Operating System}

Operating system is very important in this pen drive, because without the support of pen drive we cannot display the contents in the pen drive through display unit. The other main use of operating system in the computerized pen drive is to maintain the formats of contents in the pen drive and the transmission of contents from the computerized pen drive. The use of operating system in the pen drive will also provide the user friendly environment so that the user will feel more comfort and easy while using this computerized pen drive. We can also use the Caernarvon operating system demonstrate that a high assurance system for smart cards was technically feasible and commercially viable. The entire system has been designed to be evaluated under the Common Criteria at EAL7 [6].The processor must also be implemented for the functioning of the Computerized Pen Drive. We use the Nurture IDR segmentation and multiple instructions queues in superscalar pipelining processor [7], which is very fast and the efficient processor.

\section{F. Charge Terminals}

While using the computerized pen drives if the battery is low we can charge the pen drive by using the mobile phone adapter in the charge terminal slot. It requires only small amount of power. In the Computerized Pen Drive the charge terminals are optional while constructing of the pen drive. 


\section{DESIGNING EXTENDABLE MEMORY SLOTS}

The core idea of this proposal is creating the extendable memory slots in the body of the pen drive. This can be used in case there is a need of extra memory space. The memory can be extended by adding the memory cards in the slots provided therefore increase the space for storage. By implementing this concept the sales or the division of the pen drive devices can be brought into end.

\section{A. Reasons for Implementation of Extendable Memory}

The two main reasons which made us to design the concept of extendable memory slots in the pen drive devices are being explained as follows.

1) Insuffient memory space in the pen drive: Suppose a person is working a pen drive of memory capacity of $2 \mathrm{~GB}$ and want to store and transmit a digital file of size more than $2 \mathrm{~GB}$ may be of $3 \mathrm{~GB}$. In this case a memory card of $2 \mathrm{~GB}$ can be inserted into the pen drive in the memory slots available and the capacity of the pendrive can be extended to $4 \mathrm{~GB}$ and used for the storage of the data.

2) Enabling pen drive to act as a card reader: If the pen drive has the in-built slots for the memory cards then when the card in inserted the contents of the memory card can be read through the pen drive itself. The pen drive can act as a card reader also.

\section{B. Memory Card}

A memory card can also be called as a flash card and it is basically a type of electronic storage device based on flash memory concept for storing digital information like films, song and photos. The Memory can be used in many electronic devices like mobile phones, digital cameras and in MP3 players. It can also be used in laptop and desktop computers. The memory cards are basically small in size and rewritable storage device. The data which are stored in it will be retained even without power.

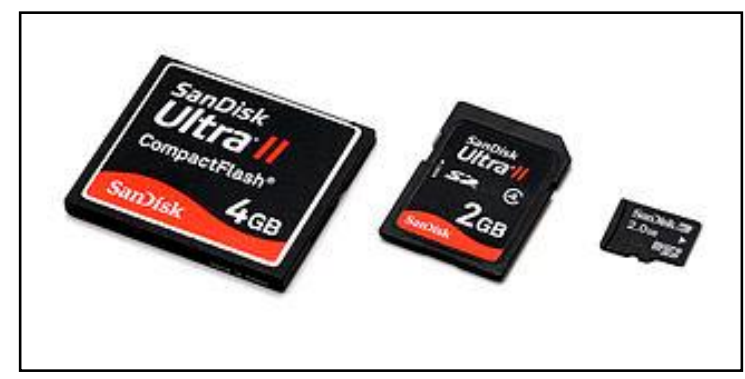

Figure 5.Types of memory cards

There are many types in the structure and the purpose of the memory cards, the different types of the memory card available in the market are listed as follows.

- $\quad$ MiniCard (Miniature Card) (max $64 \mathrm{MB}(64 \mathrm{MiB}))$

- SmartMedia Card (SSFDC) (max $128 \mathrm{MB})$ (3.3 V,5 V)

- $\quad$ PCMCIA ATA Type I Flash Memory Card

- Compact Flash Card (Type I), Compact Flash HighSpeed
- $\quad$ xD-Picture Card, xD-Picture Card Type M

- Memory Stick, MagicGate Memory Stick (max $128 \mathrm{MB})$

- Secure Digital (SD Card), Secure Digital HighSpeed, Secure Digital Plus/Xtra/etc

- $\quad$ MU-Flash (Mu-Card) (Mu-Card Alliance of OMIA)

- C-Flash

- $\quad$ SxS (S-by-S) memory card, a new memory card specification developed by SanDisk and Sony. SxS complies to the Express Card industry standard.

- NexflashWinbond Serial Flash Module (SFM) cards, size range $1 \mathrm{mb}, 2 \mathrm{mb}$ and $4 \mathrm{mb}$

\section{Structure of theMemory Card Slots}

The hardware space required for the implementation of this idea of creating the memory slots in the body of the pen drive does not occupy much space. The size of the memory card is very less. The following figure shows the structure of the memory card slots in the body of the pen drive. The inclusion of this particular feature in the pen drive makes the computerized pen drive a complete advanced model hence giving raise a new gadget for storage and for data transmission purpose. The major factor which has to be kept under consideration is the size of the pen drive device because the main advantage of these USB pen drives is that they are basically very handy and compact by implementing this feature we must not spoil the nature of the pen drives look and smart sizes. The one more important thing is the cost for implementing this features on a pen drive device must be low as possible.

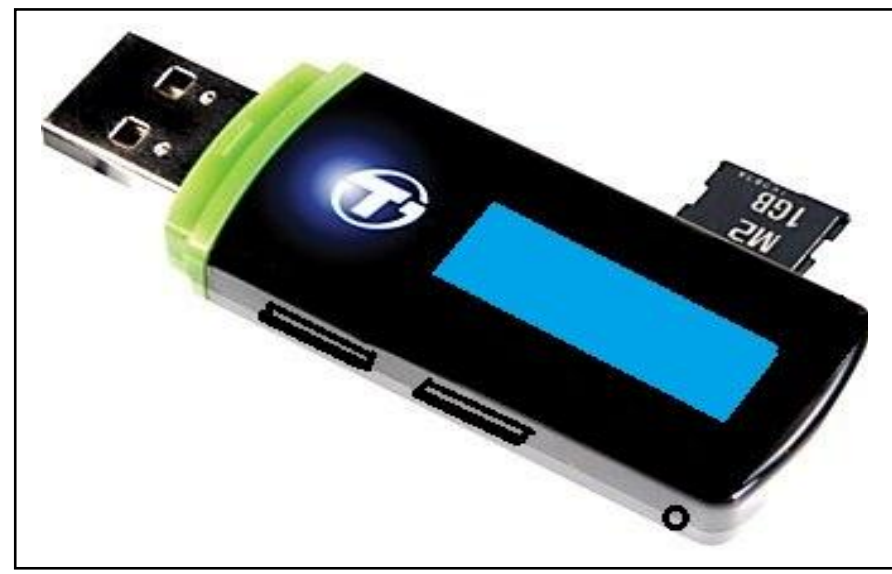

FIGURE 6. MODERN AND DIGITALIZED USB DEVICE WITH EXTENDABLE MEMORY CAPACITY

\section{CONCLUSION}

In this paper we have tried to throw light on the idea of creating a new computerized and digitalized gadget which is extremely smart and innovative. The primary goal of this proposal is to create a new pen drive model which can have an extendable memory and also be used as the card reader at the same time. Adding few features like the display screen, an operating system with the processor using the concept Nurture IDR segmentation and multiple instructions queues in 
superscalar pipelining processor, will support the transmission and also manage the contents of the pen drive and the inbuilt USB ports. The cost of the design of this pen drive is focused to cost efficient and the steps for controlling the effects against virus attack are also implemented.

\section{REFERENCES}

[1] Po-Chun Huang, Yuan-Hao Chang, Tei-Wei Kuot, Jen-Wei Hsieh, Miller Lin. May 2008, "The Behavior Analysis of Flash-Memory Storage Systems", Object Oriented Real-Time Distributed Computing (ISORC), 2008 11th IEEE International Symposium, Print ISBN: 978-07695-3132-8 J. Clerk Maxwell, A Treatise on Electricity and Magnetism, 3rd ed., vol. 2. Oxford: Clarendon, 1892, pp.68-73.

[2] Keun-Gi Lee, Hye-Won Lee, Chang-Wook Park, Je-Wan Bang, Kwonyoup Kim, Sangjin Lee, 13-15 Dec. 2008, "USB Pass On: Secure USB Thumb Drive Forensic Toolkit", Future Generation Communication and Networking Second International Conference. Print ISBN: 978-0-76953431-2.K. Elissa, "Title of paper if known," unpublished.
[3] O'Brien K, Salyers D.C, Striegel A.D, Poellabauer C, June 2008, "Power and performance characteristics of USB flash drives",World of Wireless, Mobile and Multimedia Networks International Symposium.

[4] Remple, T.B., Qualcomm, San Diego, CA, USA, June 2003 "USB onthe-go interface for portable devices", Consumer Electronics, 20 s03. ICCE. 2003, IEEE International Conference. Print ISBN: 0-7803-7721-4

[5] Seiki Takahashi, Byoung Jun Lee, Jai Hyun Koh, Satoru Saito, Bong Hyun You, Nam Deog Kim, and Sang Soo Kim, June 2009 "Embedded Liquid Crystal Capacitive Touch Screen Technology for Large Size LCD Applications", SID Symposium Digest of Technical Papers.

[6] David C. Toll, Paul A. Karger, Elaine R. Palmer, Suzanne K. McIntosh, Sam Weber, 2008 "The Caernarvon secure embedded operating system", Newsletter ACM SIGOPS Operating System.

[7] J.Nandini Meeraa, N.Indhuja, S.Devi Abirami and K.Rathinakumar, "Nurture IDR Segmentation and Multiple Instruction Queues in Superscalar Pipelining Processor", IJCSI International Journal of Computer Science Issues, Vol. 8, Issue 6, No 1, November 2011 